\title{
Decay of a model system of radiating gas
}

\author{
WENJUN WANG \\ College of Science, University of Shanghai for Science and Technology, \\ Shanghai 200093, P.R. China \\ ZHIGANG WU* \\ Department of Mathematics, Hangzhou Normal University, \\ Hangzhou 310036, P.R. China
}

\begin{abstract}
This paper is concerned with optimal time-decay estimates of solutions of the Cauchy problem to a model system of the radiating gas in $\mathbb{R}^{n}$. Compared to Liu and Kawashima (2011) [22] and Wang and Wang (2009) [33], without smallness assumption of initial perturbation in $L^{1}$-norm, we study large time behavior of small amplitude classical solutions to the Cauchy problem. The optimal $H^{N}$-norm time-decay rates of the solutions in $\mathbb{R}^{n}$ with $1 \leq n \leq 4$ are obtained by applying the Fourier splitting method introduced in Schonbek (1980) [27] with a slight modification and an energy method. Furthermore, basing on a refined pure energy method introduced in Guo and Wang [6] (2011), we give optimal $L^{p}-L^{2}\left(\mathbb{R}^{3}\right)$ decay estimates of the derivatives of solutions when initial perturbation is bounded in $L^{p}$-norm with some $p \in(1,2]$.
\end{abstract}

Key Words: the radiating gas; Fourier splitting method; negative Sobolev space; optimal time-decay rate.

\section{Introduction}

In the present paper, we consider the large time behavior of the Cauchy problem for a model system of radiating gas, taking the form of

$$
\begin{cases}u_{t}+\sum_{j=1}^{n} f_{j}(u)_{x_{j}}+\operatorname{div} q=0, & x \in \mathbb{R}^{n}, t>0, \\ -\nabla \operatorname{div} q+q+\nabla u=0, & x \in \mathbb{R}^{n}, t>0,\end{cases}
$$

with initial data

$$
u(x, 0)=u_{0}(x), \quad x \in \mathbb{R}^{n} .
$$

Here unknown functions $u=u(x, t): \mathbb{R}^{n} \times[0, \infty) \rightarrow \mathbb{R}$ and $q=q(x, t): \mathbb{R}^{n} \times[0, \infty) \rightarrow \mathbb{R}^{n}$ represent the velocity and radiating heat flux of the gas, respectively. The notations $\nabla$ and

${ }^{*}$ Corresponding author. E-mail: mathzgwu@yahoo.com.cn 
div are the $n$-dimensional gradient and divergence. $f(u)=\left(f_{1}(u), \cdots, f_{n}(u)\right) \in \mathbb{R}^{n}$ is a given smooth function of $u$ satisfying $f_{j}(u)=O\left(|u|^{2}\right)(j=1, \cdots, n)$ for $u \rightarrow 0$.

System (1.1) simplifies the model for the motion of radiating gas in several space variables. Indeed, in a certain physical situation, (1.1) is well approximated to the fundamental system describing the motion of radiating gas:

$$
\left\{\begin{array}{l}
\rho_{t}+\operatorname{div}(\rho u)=0 \\
(\rho u)_{t}+\operatorname{div}(\rho u \otimes u+p I)=0 \\
{\left[\rho\left(e+\frac{|u|^{2}}{2}\right)\right]_{t}+\operatorname{div}\left[\rho u\left(e+\frac{|u|^{2}}{2}\right)+p u+q\right]=0} \\
-\nabla \operatorname{div} q+a_{1} q+a_{2} \nabla \theta^{4}=0
\end{array}\right.
$$

where $\rho, u, p, e, \theta$ are respectively the mass density, velocity, pressure, internal energy and absolute temperature of the gas, while $q$ is the radiative heat flux. $a_{1}$ and $a_{2}$ are given positive constants depending on the gas itself. The simplified model (1.1) was first investigated by Hamer [7], and the reduction of the full system (1.3) to (1.1) was given in [5, 7, 15].

A lot of important works have been done on system (1.1). For one-dimensional case, we refer to [3, 30, 31] for $L^{1}$ stability results, [14, 16, 19] for a singular limit and relaxation limit, [11, 12, 13, 17, 18, 20, 21, 23, 24, 25] for shock waves, [8, 9, 10, for diffusion waves and [15] for rarefaction waves.

However, there are fewer studies for system (1.1) in the case of multi-dimensional space. Recently, Francesco in [2] obtained the global existence of weak entropy solutions of system (1.1) and the relaxation limits. Later, Wang and Wang in 33 investigated pointwise estimates of solutions to (1.1)-(1.2) by means of a detailed analysis for Green's function. More recently, the asymptotic decay rates toward the planar rarefaction waves based on the $L^{2}$ energy method are obtained in [4] for 2-dimensions and in [5] for $n$-dimensions $(n=3,4,5)$, respectively. The asymptotic behavior of solutions to the diffusion waves was studied in [22, 26]. For the related study of decay rates to the problem (1.1)-(1.2), we mention [22] and [1] only. 222 studied large time behavior of solutions to the problem (1.1)-(1.2) with small initial data in $L^{1}$-norm perturbation by using a time-weighted energy method. In [1], under the assumption that $\left\|u_{0}\right\|_{L^{1}}$ is bounded, Duan et al. showed that the optimal $L^{2}$-norm time-decay rate of solutions is $(1+t)^{-\frac{n}{4}}$, whereas time-decay estimates of the derivatives of the solutions have not been considered.

The purpose of this paper is to establish the optimal time decay rates of the solutions and the derivatives of the solutions to the Cauchy problem (1.1)-(1.2) without smallness assumptions on the initial data in $L^{1}$-norm. It seems that the usual energy method basing on the linearization analysis doesn't work. By the way, our first decay result is inspired from Schonbek [28, 29], where the well-known Fourier splitting method is established to get optimal decay rate of solutions for the incompressible Navier-Stokes equations in $L^{2}$-norm or $H^{s}$-norm. In the present paper, we generalize the Fourier splitting method with a slight 
modification to deal with the problem (1.1)-(1.2) for $\mathbb{R}^{n}$ with $1 \leq n \leq 4$. Our second decay result is illuminated by a recent work of Guo and Wang [6], where they developed a new method to establish optimal time decay rates of solutions to the Cauchy problem for the compressible Navier-Stokes equations and the Boltzmann equation. The main idea was to combine scaled energy estimates with an interpolation between negative and positive Sobolev norms to get the time decay rate for dissipative equations. By employing this new method, we obtain the optimal $L^{p}-L^{2}\left(\mathbb{R}^{3}\right)$ decay estimates of solutions to the problem (1.1)-(1.2).

First, we cite a temporal global existence result established in [22, 33].

Proposition 1.1.(22], Theorem 2.1; 33], Theorem 1.1) Assume that $u_{0}(x) \in H^{N}\left(\mathbb{R}^{n}\right)(n \geq$ 1) for an integer $N \geq\left[\frac{n}{2}\right]+2$. There exists a small positive constant $\delta_{0}$ such that if $E_{0}=\left\|u_{0}\right\|_{H^{N}\left(\mathbb{R}^{n}\right)} \leq \delta_{0}$, then the problem (1.1)-(1.2) has a unique global solution $(u, q)(x, t)$ satisfying

$$
\begin{aligned}
& u \in C\left([0, \infty) ; H^{N}\left(\mathbb{R}^{n}\right)\right), \nabla u \in L^{2}\left([0, \infty), H^{N-1}\left(\mathbb{R}^{n}\right)\right) \\
& q \in C\left([0, \infty) ; H^{N}\left(\mathbb{R}^{n}\right)\right) \cap L^{2}\left([0, \infty), H^{N+1}\left(\mathbb{R}^{n}\right)\right)
\end{aligned}
$$

Moreover, the solution verifies the following uniform energy estimate

$$
\|u(t)\|_{H^{N}\left(\mathbb{R}^{n}\right)}^{2}+\|q(t)\|_{H^{N+1}\left(\mathbb{R}^{n}\right)}^{2}+\int_{0}^{t}\left(\|\nabla u(\tau)\|_{H^{N-1}\left(\mathbb{R}^{n}\right)}^{2}+\|q(\tau)\|_{H^{N+1}\left(\mathbb{R}^{n}\right)}^{2}\right) \mathrm{d} \tau \leq C E_{0}^{2} .
$$

Our main results in this paper can be stated as follows:

Theorem 1.1. Let $1 \leq n \leq 4$. Suppose $\left\|u_{0}\right\|_{H^{N}\left(\mathbb{R}^{n}\right)} \leq \delta_{0} \ll 1$. If further, $u_{0} \in L^{1}\left(\mathbb{R}^{n}\right)$ (which need not be small), we have

$$
\begin{gathered}
\left\|D^{l} u(t)\right\|_{L^{2}\left(\mathbb{R}^{n}\right)} \leq C(1+t)^{-\frac{n}{4}-\frac{l}{2}}, \quad l=0,1, \cdots, N ; \\
\left\|D^{l} q(t)\right\|_{L^{2}\left(\mathbb{R}^{n}\right)} \leq C(1+t)^{-\frac{n}{4}-\frac{l+1}{2}}, \quad l=1,2, \cdots, N-1 .
\end{gathered}
$$

Remark 1.1. One can rewrite the system (1.1) as a decouple system of $(u, q)(x, t)$ :

$$
\left\{\begin{array}{l}
u_{t}+\sum_{j=1}^{n} f_{j}(u)_{x_{j}}=-u+(I-\Delta)^{-1} u \\
q=-(I-\Delta)^{-1} \nabla u
\end{array}\right.
$$

To obtain the time-decay rates of $q(x, t)$, from $(1.7)_{2}$, it suffices to prove the estimates on $u(x, t)$, i.e. (1.5). When $l=0,(1.5)$ can be obtained directly by using the usual Fourier splitting method. In addition, for any integer $n \geq 1$, (1.5) also holds true, see Proposition 3.1 in Section 3. This is consistent with the result in [1]. When $l \geq 1$, to prove (1.5), the estimates in the Proposition 1.1 and the smallness of $E_{0}$ should be employed, see details in the proof of Proposition 3.2. 
Theorem 1.2. Suppose $\left\|u_{0}\right\|_{H^{N}\left(\mathbb{R}^{3}\right)} \leq \delta_{0} \ll 1$. If further, $u_{0} \in \dot{H}^{-s}\left(\mathbb{R}^{3}\right)$ (which need not be small), for some $s \in\left[0, \frac{3}{2}\right)$, there exists a positive constant $C_{0}$ such that

$$
\|u(t)\|_{\dot{H}^{-s}\left(\mathbb{R}^{3}\right)}^{2} \leq C_{0}, \quad\|q(t)\|_{\dot{H}^{-s}\left(\mathbb{R}^{3}\right)}^{2} \leq C_{0},
$$

and the following decay estimates hold:

$$
\begin{gathered}
\left\|D^{l} u(t)\right\|_{H^{N-l}\left(\mathbb{R}^{3}\right)} \leq C_{0}(1+t)^{-\frac{l+s}{2}}, l=0, \cdots, N, \\
\left\|D^{l} q(t)\right\|_{H^{N-1-l}\left(\mathbb{R}^{3}\right)} \leq C_{0}(1+t)^{-\frac{l+s+1}{2}}, l=0, \cdots, N-1 .
\end{gathered}
$$

By employing the Hardy-littlewood-Sobolev theorem, for $p \in(1,2]$, we have $L^{p} \in$ $\dot{H}^{-s}\left(\mathbb{R}^{3}\right)$ with $s=3\left(\frac{1}{p}-\frac{1}{2}\right) \in\left[0, \frac{3}{2}\right)$. Then, from Theorem 1.2 , the following optimal $L^{p}-L^{2}$ type decay results are obtained.

Corollary 1.1. ( $L^{p}-L^{2}$ time-decay estimates) Assume that $\left\|u_{0}\right\|_{H^{N}\left(\mathbb{R}^{3}\right)} \leq \delta_{0} \ll 1$. If further, $u_{0}(x) \in L^{p}\left(\mathbb{R}^{3}\right)$ with some $p \in(1,2]$, then the following decay results hold for any integer $l$ with $0 \leq l \leq N$ :

$$
\begin{aligned}
\left\|D^{l} u(t)\right\|_{H^{N-l}\left(\mathbb{R}^{3}\right)} & \leq C_{0}(1+t)^{-\frac{3}{2}\left(\frac{1}{p}-\frac{1}{2}\right)-\frac{l}{2}} . \\
\left\|D^{l} q(t)\right\|_{H^{N+1-l}\left(\mathbb{R}^{3}\right)} & \leq C_{0}(1+t)^{-\frac{3}{2}\left(\frac{1}{p}-\frac{1}{2}\right)-\frac{l+1}{2}} .
\end{aligned}
$$

Remark 1.2. All the decay results above are obtained without the smallness of the initial perturbation in $L^{p}\left(\mathbb{R}^{3}\right), p \in(1,2]$ or $\dot{H}^{-s}\left(\mathbb{R}^{3}\right)$. The results generalize those in [22, 33] for the case of three-dimensional space. The similar problem in $\mathbb{R}^{n}$ with $n=1,2$ will be investigated in future.

Notations. In this paper, $D^{l}$ with an integer $l \geq 0$ stands for the usual any spatial derivatives of order $l$. For $1 \leq p \leq \infty$ and an integer $m \geq 0$, we use $L^{p}$ and $W^{m, p}$ denote the usual Lebesgue space $L^{p}\left(\mathbb{R}^{n}\right)$ and Sobolev spaces $W^{m, p}\left(\mathbb{R}^{n}\right)$ with norms $\|\cdot\|_{L^{p}}$ and $\|\cdot\|_{W^{m, p}}$, respectively, and set $H^{m}=W^{m, 2}$ with norm $\|\cdot\|_{H^{m}}$ when $p=2$. In addition, for $s \in \mathbb{R}$, we define a pseudo-differential operator $\Lambda^{s}$ by

$$
\Lambda^{s} g(x)=\int_{\mathbb{R}^{n}}|\xi|^{s} \hat{g}(\xi) \mathrm{e}^{2 \pi \sqrt{-1} x \cdot \xi} \mathrm{d} \xi
$$

where $\hat{g}$ denotes the Fourier transform of $g$. We define the homogeneous Sobolev space $\dot{H}^{s}$ of all $g$ for which $\|g\|_{\dot{H}^{s}}$ is finite, where

$$
\|g\|_{\dot{H}^{s}}:=\left\|\Lambda^{s} g\right\|_{L^{2}}=\left\||\xi|^{s} \hat{g}\right\|_{L^{2}} .
$$

Throughout this paper, we will use a non-positive index $s$. For convenience, we will change the index to be " $-s$ " with $s \geq 0$. $C$ denotes a positive generic (generally large) constant that may vary at different places. The integration domain $\mathbb{R}^{3}$ will be always omitted without any ambiguity. 
The rest of this paper is arranged as follows. In the next section, some Sobolev type inequalities and some preliminaries are given for later use. Section 3 shows the proof of Theorem 1.1 by using Fourier splitting method. In the last section, we obtain the time-decay estimates stated in Theorem 1.2.

\section{Preliminaries}

Firstly, we give some Sobolev inequalities which will be used in the next two sections.

Lemma 2.1. (Gagliardo-Nirenberg's inequality). Let $0 \leq m, k \leq l$, then we have

$$
\left\|D^{k} g\right\|_{L^{p}} \leq C\left\|D^{m} g\right\|_{L^{q}}^{1-\theta}\left\|D^{l} g\right\|_{L^{r}}^{\theta}
$$

where $k$ satisfies

$$
\frac{1}{p}-\frac{k}{n}=(1-\theta)\left(\frac{1}{q}-\frac{m}{n}\right)+\theta\left(\frac{1}{r}-\frac{l}{n}\right) .
$$

Lemma 2.2. ([ $[6]$, Lemma A.5) Let $s \geq 0$ and $l \geq 0$, then we have

$$
\left\|D^{l} g\right\|_{L^{2}} \leq C\left\|D^{l+1} g\right\|_{L^{2}}^{1-\theta}\|g\|_{\dot{H}^{-s}}^{\theta}, \text { where } \theta=\frac{1}{l+s+1} .
$$

Lemma 2.3. ([32], Chapter V, Theorem 1) Let $0<s<n, 1<p<q<\infty, \frac{1}{q}+\frac{s}{n}=\frac{1}{p}$, then

$$
\left\|\Lambda^{-s} g\right\|_{L^{q}} \leq C\|g\|_{L^{p}}
$$

Now, when $n=3$, we derive an estimate of Lyapunov-type which plays an important role in closing the energy estimates at each $l$-th level in Section 4.

Proposition 2.1. Let $(u, q)(x, t)$ be a solution to the Cauchy problem (1.1)-(1.2) in $\mathbb{R}^{3}$. If the assumptions in Proposition 1.1 hold, we have

$$
\frac{\mathrm{d}}{\mathrm{d} t}\left\|D^{l} u(t)\right\|_{H^{1}}^{2}+\left\|D^{l+1} u(t)\right\|_{L^{2}}^{2} \leq 0, \quad 0 \leq l \leq N-1 .
$$

Proof. First, we transform system (1.1) into the following equivalent decoupled system

$$
\begin{cases}u_{t}-\Delta u_{t}-\Delta u=-(1-\Delta) \sum_{j=1}^{n} f_{j}(u)_{x_{j}}, & x \in \mathbb{R}^{n}, t>0, \\ q=-(1-\Delta)^{-1} u, & x \in \mathbb{R}^{n}, t>0 .\end{cases}
$$

Multiplying $(2.2)_{1}$ by $u$, then a direct calculation gives

$$
\begin{aligned}
& \left(u^{2}+|\nabla u|^{2}\right)_{t}+2|\nabla u|^{2}-2 \operatorname{div}\left[u \nabla\left(u_{t}+u+\sum_{j=1}^{3} f_{j}(u)_{x_{j}}\right)\right]+\sum_{j=1}^{3}\left[2 \int_{0}^{u} f_{j}^{\prime}(\eta) \eta d \eta\right]_{x_{j}} \\
= & -\sum_{j=1}^{3} f_{j}^{\prime \prime}(u) u_{x_{j}}|\nabla u|^{2} .
\end{aligned}
$$


Integrating the above equality with respect to $x$ on $\mathbb{R}^{3}$ and using Lemma 2.1, we get

$$
\frac{\mathrm{d}}{\mathrm{d} t}\|u(t)\|_{H^{1}}^{2}+\|D u(t)\|_{L^{2}}^{2} \leq C\|\nabla u(t)\|_{L^{\infty}}\|D u(t)\|_{L^{2}}^{2} .
$$

As a result, we find

$$
\frac{\mathrm{d}}{\mathrm{d} t}\|u(t)\|_{H^{1}}^{2}+\|D u(t)\|_{L^{2}}^{2} \leq 0
$$

Since $f_{j}(u)=O\left(u^{2}\right)$ when $u \rightarrow 0$, without loss of generality, let $f_{j}(u)=u^{2}$. In terms of estimates for the derivatives of the solution $u$, one can apply $D^{l}$ on $(2.2)_{1}$, and multiply the resulting equality by $D^{l} u$, then integrating it with respect to $x$ over $\mathbb{R}^{3}$, obtaining

$$
\begin{aligned}
& \frac{\mathrm{d}}{\mathrm{d} t}\left(\left\|D^{l} u(t)\right\|_{L^{2}}^{2}+\left\|\nabla D^{l} u(t)\right\|_{L^{2}}^{2}\right)+2\left\|\nabla D^{l} u(t)\right\|_{L^{2}}^{2} \\
= & -\sum_{j=1}^{3} \int D^{l}\left(u u_{x_{j}}\right) D^{l} u \mathrm{~d} x-\sum_{j=1}^{3} \int D^{l} \nabla\left(u u_{x_{j}}\right) D^{l} \nabla u \mathrm{~d} x=: I_{1}+I_{2} .
\end{aligned}
$$

For $I_{1}$, by using Hölder's inequality and Lemma 2.1, we have

$$
\begin{aligned}
I_{1} & =-\sum_{j=1}^{3} \int_{\mathbb{R}^{3}} D^{l}\left(u u_{x_{j}}\right) D^{l} u \mathrm{~d} x=-\sum_{j=1}^{3} \sum_{0 \leq k \leq l} \int_{\mathbb{R}^{3}}\left(D^{k} u D^{l-k} u_{x_{j}}\right) D^{l} u \mathrm{~d} x \\
& \leq C \sum_{0 \leq k \leq l}\left\|\left(D^{k} u D^{l-k+1} u\right)(t)\right\|_{L^{\frac{6}{5}}}\left\|D^{l} u(t)\right\|_{L^{6}} \\
& \leq C \sum_{0 \leq k \leq l}\left\|\left(D^{k} u D^{l-k+1} u\right)(t)\right\|_{L^{\frac{6}{5}}}\left\|D^{l+1} u(t)\right\|_{L^{2}} .
\end{aligned}
$$

When $k \leq\left[\frac{l}{2}\right]$, by using Hölder's inequality and Lemma 2.1, we get

$$
\begin{aligned}
\left\|\left(D^{k} u D^{l-k+1} u\right)(t)\right\|_{L^{\frac{6}{5}}} & \leq C\left\|D^{k} u(t)\right\|_{L^{3}}\left\|D^{l-k+1} u(t)\right\|_{L^{2}} \\
& \leq C\left\|D^{m} u(t)\right\|_{L^{2}}^{1-\frac{k}{l+1}}\left\|D^{l+1} u(t)\right\|_{L^{2}}^{\frac{k}{l+1}}\|u(t)\|_{L^{2}}^{\frac{k}{l+1}}\left\|D^{l+1} u(t)\right\|_{L^{2}}^{1-\frac{k}{l+1}} \\
& \leq C\left(\left\|D^{m} u(t)\right\|_{L^{2}}+\|u(t)\|_{L^{2}}\right)\left\|D^{l+1} u(t)\right\|_{L^{2}} \leq \frac{1}{12}\left\|D^{l+1} u(t)\right\|_{L^{2}},
\end{aligned}
$$

where we have used the fact $\|u(t)\|_{H^{N}} \leq C E_{0}^{2} \ll 1$ in Proposition 1.1, and $m$ satisfies

$$
\frac{k}{3}-\frac{1}{3}=\left(\frac{m}{3}-\frac{1}{2}\right) \times\left(1-\frac{k}{l+1}\right)+\left(\frac{l+1}{3}-\frac{1}{2}\right) \times \frac{k}{l+1} .
$$

As a result, since $k \leq\left[\frac{l}{2}\right]$, we have

$$
m=\frac{l+1}{2(l+1-k)} \in\left[\frac{1}{2}, 1\right) .
$$

When $k \geq\left[\frac{l}{2}\right]+1$, from Hölder's inequality and Lemma 2.1 again, we find

$$
\begin{aligned}
\left\|\left(D^{k} u D^{l+1-k} u\right)(t)\right\|_{L^{\frac{6}{5}}} & \leq C\left\|D^{k} u(t)\right\|_{L^{2}}\left\|D^{l+1-k} u(t)\right\|_{L^{3}} \\
& \leq C\|u(t)\|_{L^{2}}^{1-\frac{k}{l+1}}\left\|D^{l+1} u(t)\right\|_{L^{2}}^{\frac{k}{l+1}}\left\|D^{m} u(t)\right\|_{L^{2}}^{\frac{k}{l+1}}\left\|D^{l+1} u(t)\right\|_{L^{2}}^{1-\frac{k}{l+1}} \\
& \leq C\left(\left\|D^{m} u(t)\right\|_{L^{2}}+\|u(t)\|_{L^{2}}\right)\left\|D^{l+1} u(t)\right\|_{L^{2}} \leq \frac{1}{4}\left\|D^{l+1} u(t)\right\|_{L^{2}},
\end{aligned}
$$


where $m$ is defined by

$$
\frac{l+1-k}{3}-\frac{1}{3}=\left(\frac{m}{3}-\frac{1}{2}\right) \times \frac{k}{l+1}+\left(\frac{l+1}{3}-\frac{1}{2}\right) \times\left(1-\frac{k}{l+1}\right),
$$

that is, $m=\frac{l+1}{2 k} \in\left(\frac{1}{2}, 1\right]$ since $k \geq \frac{l+1}{2}$.

From (2.5), (2.6) and (2.8), we have

$$
I_{1}=-\sum_{j=1}^{3} \int D^{l}\left(u u_{x_{j}}\right) D^{l} u \mathrm{~d} x \leq \frac{1}{4}\left\|D^{l+1} u(t)\right\|_{L^{2}} .
$$

The estimate of $I_{2}$ is absolutely the same to (3.10)-(3.12) except that we replace $l$ by $l+1$. In fact, we have

$$
I_{2}=-\sum_{j=1}^{3} \int D^{l} \nabla\left(u u_{x_{j}}\right) D^{l} \nabla u \mathrm{~d} x \leq \frac{1}{4}\left\|D^{l+1} u(t)\right\|_{L^{2}} .
$$

Thus, from (2.4), (2.9) and (2.10), we get the estimate of Lyaponov-type (2.1). Then, we complete the proof of Proposition 2.1.

\section{Decay results with initial perturbation in $L^{1}\left(\mathbb{R}^{n}\right)$}

In this section, we will give optimal decay results by Fourier splitting method introduced in [27, 28] together with energy estimates. Theorem 1.1 will be proved by the following lemmas. First, a straightforward application of Fourier splitting method yields an optimal $L^{2}$-norm time-decay rates of solutions as follows.

Proposition 3.1. If the initial data $u_{0}(x) \in L^{1}\left(\mathbb{R}^{n}\right) \cap L^{2}\left(\mathbb{R}^{n}\right)$ with $n \geq 1$, one has

$$
\|u(t)\|_{L^{2}\left(\mathbb{R}^{n}\right)}^{2} \leq C\left(\left\|u_{0}\right\|_{L^{1}\left(\mathbb{R}^{n}\right)}+\left\|u_{0}\right\|_{L^{2}\left(\mathbb{R}^{n}\right)}\right)(1+t)^{-\frac{n}{2}} .
$$

Proof. First, multiplying $(1.7)_{1}$ by $u$ and summing up them and then integrating over $\mathbb{R}^{n}$, we obtain

$$
\begin{aligned}
\frac{1}{2} \frac{\mathrm{d}}{\mathrm{d} t} \int_{\mathbb{R}^{n}} u^{2} \mathrm{~d} x & =\int_{\mathbb{R}^{n}} \sum_{j=1}^{n} f_{j}(u) \partial_{x_{j}} u \mathrm{~d} x-\int_{\mathbb{R}^{n}} u^{2} \mathrm{~d} x+\int_{\mathbb{R}^{n}} u(1-\Delta)^{-1} u \mathrm{~d} x \\
& =-\int_{\mathbb{R}^{n}} u^{2} \mathrm{~d} x+\int_{\mathbb{R}^{n}} u(1-\Delta)^{-1} u \mathrm{~d} x .
\end{aligned}
$$

By Plancherel theorem, we get

$$
\begin{aligned}
& \frac{\mathrm{d}}{\mathrm{d} t} \int_{\mathbb{R}^{n}}|\hat{u}|^{2} \mathrm{~d} \xi=-\int_{\mathbb{R}^{n}}|\hat{u}|^{2} \mathrm{~d} \xi+\int_{\mathbb{R}^{n}} \frac{1}{1+|\xi|^{2}}|\hat{u}|^{2} \mathrm{~d} \xi \\
= & -\int_{\mathbb{R}^{n}}|\hat{u}|^{2} \mathrm{~d} \xi+\int_{|\xi| \leq \sqrt{\frac{n}{t}}} \frac{1}{1+|\xi|^{2}}|\hat{u}|^{2} \mathrm{~d} \xi+\int_{|\xi|>\sqrt{\frac{n}{t}}} \frac{1}{1+|\xi|^{2}}|\hat{u}|^{2} \mathrm{~d} \xi \\
\leq & -\int_{\mathbb{R}^{n}}|\hat{u}|^{2} \mathrm{~d} \xi+\int_{|\xi|>\sqrt{\frac{n}{t}}} \frac{t}{n+t}|\hat{u}|^{2} \mathrm{~d} \xi+\int_{|\xi| \leq \sqrt{\frac{n}{t}}} \frac{1}{1+|\xi|^{2}}|\hat{u}|^{2} \mathrm{~d} \xi \\
= & -\frac{n}{n+t} \int_{\mathbb{R}^{n}}|\hat{u}|^{2} \mathrm{~d} \xi+\int_{|\xi| \leq \sqrt{\frac{n}{t}}} \frac{1}{1+|\xi|^{2}}|\hat{u}|^{2} \mathrm{~d} \xi-\frac{t}{n+t} \int_{|\xi| \leq \sqrt{\frac{n}{t}}}|\hat{u}|^{2} \mathrm{~d} \xi .
\end{aligned}
$$


We rewrite (3.2) as follows.

$$
\frac{\mathrm{d}}{\mathrm{d} t} \int_{\mathbb{R}^{n}}|\hat{u}|^{2} \mathrm{~d} \xi+\frac{n}{n+t} \int_{\mathbb{R}^{n}}|\hat{u}|^{2} \mathrm{~d} \xi \leq \int_{|\xi| \leq \sqrt{\frac{n}{t}}}\left(\frac{1}{1+|\xi|^{2}}-\frac{t}{n+t}\right)|\hat{u}|^{2} \mathrm{~d} \xi,
$$

which is multiplied by $(n+t)^{n}$ yields

$$
\begin{aligned}
\frac{\mathrm{d}}{\mathrm{d} t}\left[(n+t)^{n} \int_{\mathbb{R}^{n}}|\hat{u}|^{2} \mathrm{~d} \xi\right] & \leq(n+t)^{n} \int_{|\xi| \leq \sqrt{\frac{n}{t}}}\left(\frac{1}{1+|\xi|^{2}}-\frac{t}{n+t}\right)|\hat{u}|^{2} \mathrm{~d} \xi \\
& \leq(n+t)^{n}\|\hat{u}(t)\|_{L^{\infty}\left(\mathbb{R}_{\xi}^{n}\right)}^{2} \int_{|\xi| \leq \sqrt{\frac{n}{t}}}\left(1-\frac{t}{n+t}\right) \mathrm{d} \xi \\
& \leq C\|u(t)\|_{L^{1}\left(\mathbb{R}^{n}\right)}^{2}(n+t)^{n-1}(n+t)^{-\frac{n}{2}} \\
& \leq C\left\|u_{0}\right\|_{L^{1}\left(\mathbb{R}^{n}\right)}^{2}(n+t)^{\frac{n}{2}-1},
\end{aligned}
$$

where we have used $\|\hat{u}(t)\|_{L^{\infty}\left(\mathbb{R}_{\xi}^{n}\right)} \leq\|u(t)\|_{L^{1}\left(\mathbb{R}^{n}\right)}$ and the fact $\|u(t)\|_{L^{1}\left(\mathbb{R}^{n}\right)} \leq\left\|u_{0}\right\|_{L^{1}\left(\mathbb{R}^{n}\right)}$ in [2, 4, 5].

Integrating (3.3) with respect to $t$, we have

$$
\|u(t)\|_{L^{2}\left(\mathbb{R}^{n}\right)}^{2} \leq C\left(\left\|u_{0}\right\|_{L^{1}\left(\mathbb{R}^{n}\right)}+\left\|u_{0}\right\|_{L^{2}\left(\mathbb{R}^{n}\right)}\right)(n+t)^{-\frac{n}{2}}
$$

This proves Proposition 3.1.

In the proof of Lemma 2.1, we used the essential fact that $\int_{\mathbb{R}^{n}} \sum_{j=1}^{n} f_{j}(u) u_{x_{j}} \mathrm{~d} x$ in (3.1) is equal to 0 . However, for the derivatives of $u$, the Fourier splitting method above can not be used directly. The following is the main reason. Similar to prove (3.1), we have

$$
\frac{1}{2} \frac{\mathrm{d}}{\mathrm{d} t} \int_{\mathbb{R}^{n}}\left|D^{l} u\right|^{2} \mathrm{~d} x=\int_{\mathbb{R}^{n}} \sum_{j=1}^{n} D^{l} f_{j}(u)_{x_{j}} D^{l} u \mathrm{~d} x-\int_{\mathbb{R}^{n}}\left|D^{l} u\right|^{2} \mathrm{~d} x+\int_{\mathbb{R}^{n}} D^{l} u(1-\Delta)^{-1} D^{l} u \mathrm{~d} x .
$$

But, the term $\int_{\mathbb{R}^{n}} \sum_{j=1}^{n} D^{l} f_{j}(u)_{x_{j}} D^{l} u \mathrm{~d} x$ in the RHS of (3.5) is not equal to 0. How to control this term? Here we use the existence results and the smallness of solutions in $H^{N}$ space stated in Proposition 1.1.

Proposition 3.2. Let $1 \leq n \leq 4$. Suppose $\left\|u_{0}\right\|_{H^{N}\left(\mathbb{R}^{n}\right)} \ll 1$. If $u_{0}(x) \in L^{1}\left(\mathbb{R}^{n}\right)$ and $D^{l} u_{0}(x) \in L^{2}\left(\mathbb{R}^{n}\right)$, one has

$$
\left\|D^{l} u(t)\right\|_{L^{2}\left(\mathbb{R}^{n}\right)}^{2} \leq C\left(\left\|u_{0}\right\|_{L^{1}\left(\mathbb{R}^{n}\right)}+\left\|D^{l} u_{0}\right\|_{L^{2}\left(\mathbb{R}^{n}\right)}\right)(1+t)^{-\frac{n}{2}-l}, l=1,2, \cdots, N .
$$

Proof. Applying $D^{l}(1 \leq l \leq N)$ on $(1.7)_{1}$, and multiplying the resulting equality by $D^{l} u$, then integrating it with respect to $x$ over $\mathbb{R}^{n}$, one has

$$
\frac{1}{2} \frac{\mathrm{d}}{\mathrm{d} t} \int_{\mathbb{R}^{n}}\left|D^{l} u\right|^{2} \mathrm{~d} x=-\int_{\mathbb{R}^{n}} \sum_{j=1}^{n} D^{l} f_{j}(u)_{x_{j}} D^{l} u \mathrm{~d} x-\int_{\mathbb{R}^{n}}\left|D^{l} u\right|^{2} \mathrm{~d} x+\int_{\mathbb{R}^{n}} D^{l} u(1-\Delta)^{-1} D^{l} u \mathrm{~d} x
$$


As mentioned above, to use the Fourier splitting method as Proposition 3.1, we have to get

$$
\left|\int_{\mathbb{R}^{n}} \sum_{j=1}^{n} D^{l} f_{j}(u)_{x_{j}} D^{l} u \mathrm{~d} x\right| \leq \delta_{0} \int_{\mathbb{R}^{n}}\left|D^{l} u\right|^{2} \mathrm{~d} x,
$$

where $0 \leq \delta_{0} \ll 1$. In the following, we only prove (3.8) for $3 \leq l \leq N$. For $l=1,2$, the proof of $(3.8)$ is similar. We omit it here. Without loss of generality, let $f_{j}(u)=u^{2}$, one has

$$
\begin{aligned}
& \int_{\mathbb{R}^{n}} D^{l} f_{j}(u)_{x_{j}} D^{l} u \mathrm{~d} x \\
& =\int_{\mathbb{R}^{n}} u D^{l} u_{x_{j}} D^{l} u \mathrm{~d} x+\int_{\mathbb{R}^{n}} D u D^{l-1} u_{x_{j}} D^{l} u \mathrm{~d} x+\sum_{2 \leq k \leq(l-1)} \int_{\mathbb{R}^{n}} C_{k}^{l} D^{k} u D^{l+1-k} u D^{l} u \mathrm{~d} x \\
& \leq 2\|D u(t)\|_{L^{\infty}\left(\mathbb{R}^{n}\right)}\left\|D^{l} u(t)\right\|_{L^{2}\left(\mathbb{R}^{n}\right)}^{2}+C \sum_{2 \leq k \leq(l-1)} \int_{\mathbb{R}^{n}} D^{k} u D^{l+1-k} u D^{l} u \mathrm{~d} x \\
& \leq 2\|D u(t)\|_{L^{\infty}\left(\mathbb{R}^{n}\right)}\left\|D^{l} u(t)\right\|_{L^{2}\left(\mathbb{R}^{n}\right)}^{2}+C\left\|D^{k} u(t)\right\|_{L^{4}\left(\mathbb{R}^{n}\right)}\left\|D^{l-k} u_{x_{j}}(t)\right\|_{L^{4}\left(\mathbb{R}^{n}\right)}\left\|D^{l} u(t)\right\|_{L^{2}\left(\mathbb{R}^{n}\right)} \\
& \leq 2\|D u(t)\|_{L^{\infty}\left(\mathbb{R}^{n}\right)}\left\|D^{l} u(t)\right\|_{L^{2}\left(\mathbb{R}^{n}\right)}^{2} \\
& +C\|u(t)\|_{L^{2}\left(\mathbb{R}^{n}\right)}^{1-\theta_{1}}\left\|D^{l} u(t)\right\|_{L^{2}\left(\mathbb{R}^{n}\right)}^{\theta_{1}}\|u(t)\|_{L^{2}\left(\mathbb{R}^{n}\right)}^{1-\theta_{2}}\left\|D^{l} u(t)\right\|_{L^{2}\left(\mathbb{R}^{n}\right)}^{\theta_{2}}\left\|D^{l} u(t)\right\|_{L^{2}\left(\mathbb{R}^{n}\right)} \\
& \leq 2\|D u(t)\|_{L^{\infty}\left(\mathbb{R}^{n}\right)}\left\|D^{l} u(t)\right\|_{L^{2}\left(\mathbb{R}^{n}\right)}^{2}+C\|u(t)\|_{L^{2}\left(\mathbb{R}^{n}\right)}^{2-\left(\theta_{1}+\theta_{2}\right)}\left\|D^{l} u(t)\right\|_{L^{2}\left(\mathbb{R}^{n}\right)}^{\theta_{1}+\theta_{2}+1} \\
& =2\|D u(t)\|_{L^{\infty}\left(\mathbb{R}^{n}\right)}\left\|D^{l} u(t)\right\|_{L^{2}\left(\mathbb{R}^{n}\right)}^{2}+C\|u(t)\|_{L^{2}\left(\mathbb{R}^{n}\right)}^{2-\left(\theta_{1}+\theta_{2}\right)}\left\|D^{l} u(t)\right\|_{L^{2}\left(\mathbb{R}^{n}\right)}^{\theta_{1}+\theta_{2}-1}\left\|D^{l} u(t)\right\|_{L^{2}\left(\mathbb{R}^{n}\right)}^{2},
\end{aligned}
$$

where $C_{k}^{l}=\left(\begin{array}{l}l \\ k\end{array}\right)$ and

$$
\theta_{1}=\frac{k+\frac{n}{4}}{l} \text { and } \theta_{2}=\frac{l-k+\frac{n}{4}+1}{l}
$$

By noticing $1 \leq n \leq 4$ and $2 \leq k \leq l-1$, from (3.10), we know $0<\theta_{1} \leq 1$ and $0<\theta_{2} \leq 1$. Then, it follows from (3.9) and the fact $\|u(t)\|_{H^{N}} \ll 1$ that

$$
\int_{\mathbb{R}^{n}} D^{l} f_{j}(u)_{x_{j}} D^{l} u \mathrm{~d} x \leq \frac{1}{2}\left\|D^{l} u(t)\right\|_{L^{2}\left(\mathbb{R}^{n}\right)}^{2} .
$$

Combining (3.11) and (3.7), we have

$$
\frac{\mathrm{d}}{\mathrm{d} t} \int_{\mathbb{R}^{n}}\left(D^{l} u\right)^{2} \mathrm{~d} x \leq-\int_{\mathbb{R}^{n}}\left(D^{l} u\right)^{2} \mathrm{~d} x+\int_{\mathbb{R}^{n}} D^{l} u(1-\Delta)^{-1} D^{l} u \mathrm{~d} x .
$$

From Plancherel theorem, one has

$$
\begin{aligned}
& \left.\left.\frac{\mathrm{d}}{\mathrm{d} t} \int_{\mathbb{R}^{n}}|| \xi\right|^{2 l} \hat{u}\right|^{2} \mathrm{~d} \xi \leq-\left.\left.\int_{\mathbb{R}^{n}}|| \xi\right|^{2 l} \hat{u}\right|^{2} \mathrm{~d} \xi+\left.\left.\int_{\mathbb{R}^{n}} \frac{1}{1+|\xi|^{2}}|| \xi\right|^{2 l} \hat{u}\right|^{2} \mathrm{~d} \xi \\
\leq & -\left.\left.\int_{\mathbb{R}^{n}}|| \xi\right|^{2 l} \hat{u}\right|^{2} \mathrm{~d} \xi+\left.\left.\int_{|\xi|>\sqrt{\frac{n+2 l}{t}}} \frac{t}{n+2 l+t}|| \xi\right|^{2 l} \hat{u}\right|^{2} \mathrm{~d} \xi+\left.\left.\int_{|\xi| \leq \sqrt{\frac{n+2 l}{t}}} \frac{1}{1+|\xi|^{2}}|| \xi\right|^{2 l} \hat{u}\right|^{2} \mathrm{~d} \xi \\
\leq & -\left.\left.\frac{n+2 l}{n+2 l+t} \int_{\mathbb{R}^{n}}|| \xi\right|^{2 l} \hat{u}\right|^{2} \mathrm{~d} \xi+\left.\left.\int_{|\xi| \leq \sqrt{\frac{n+2 l}{t}}} \frac{1}{1+|\xi|^{2}}|| \xi\right|^{2 l} \hat{u}\right|^{2} \mathrm{~d} \xi-\left.\frac{t}{n+2 l+t} \int_{|\xi| \leq \sqrt{\frac{n+2 l}{t}}}|\xi|^{2 l} \hat{u}\right|^{2} \mathrm{~d} \xi .
\end{aligned}
$$


We rewrite (3.13) as

$$
\begin{aligned}
& \left.\left.\frac{\mathrm{d}}{\mathrm{d} t} \int_{\mathbb{R}^{n}}|| \xi\right|^{2 l} \hat{u}\right|^{2} \mathrm{~d} \xi+\left.\left.\frac{n+2 l}{n+2 l+t} \int_{\mathbb{R}^{n}}|| \xi\right|^{2 l} \hat{u}\right|^{2} \mathrm{~d} \xi \\
\leq & \left.\left.\int_{|\xi| \leq \sqrt{\frac{n+2 l}{t}}}\left(\frac{1}{1+|\xi|^{2}}-\frac{t}{n+2 l+t}\right)|| \xi\right|^{2 l} \hat{u}\right|^{2} \mathrm{~d} \xi .
\end{aligned}
$$

Consequently, multiplying (3.14) by $(n+2 l+t)^{n+2 l}$, one has

$$
\begin{aligned}
& \frac{\mathrm{d}}{\mathrm{d} t}\left[\left.\left.(n+2 l+t)^{n+2 l} \int_{\mathbb{R}^{n}}|| \xi\right|^{2 l} \hat{u}\right|^{2} \mathrm{~d} \xi\right] \\
\leq & \left.\left.(n+2 l+t)^{n+2 l} \int_{|\xi| \leq \sqrt{\frac{n+2 l}{t}}}\left(\frac{1}{1+|\xi|^{2}}-\frac{t}{n+2 l+t}\right)|| \xi\right|^{2 l} \hat{u}\right|^{2} \mathrm{~d} \xi \\
\leq & (n+2 l+t)^{n+2 l}\|\hat{u}(t)\|_{L^{\infty}\left(\mathbb{R}_{\xi}^{n}\right)}^{2} \int_{|\xi| \leq \sqrt{\frac{n+2 l}{t}}}\left(1-\frac{t}{n+2 l+t}\right)|\xi|^{2 l} \mathrm{~d} \xi \\
\leq & C\|u(t)\|_{L^{1}\left(\mathbb{R}^{n}\right)}^{2}(n+2 l+t)^{n+2 l-1}(n+2 l+t)^{-\frac{n+2 l}{2}} \\
\leq & C\left\|u_{0}\right\|_{L^{1}\left(\mathbb{R}^{n}\right)}^{2}(n+2 l+t)^{\frac{n+2 l}{2}-1} .
\end{aligned}
$$

Integrating (3.15) with respect to $t$ over $(0, t)$, we find

$$
\left\|D^{l} u(t)\right\|_{L^{2}\left(\mathbb{R}^{n}\right)}^{2}=\left\||\xi|^{l} \hat{u}(t)\right\|_{L^{2}\left(\mathbb{R}_{\xi}^{n}\right)}^{2} \leq C\left(\left\|u_{0}\right\|_{L^{1}\left(\mathbb{R}^{n}\right)}+\left\|D^{l} u_{0}\right\|_{L^{2}\left(\mathbb{R}^{n}\right)}\right)(n+2 l+t)^{-\frac{n+2 l}{2}} .
$$

Then, we get (3.6).

\section{Decay results with initial perturbation in $\dot{H}^{-s}\left(\mathbb{R}^{3}\right)$}

This section devotes to the optimal $L^{p}-L^{2}\left(\mathbb{R}^{3}\right)$ decay rates of solutions to (1.1)-(1.2) when the initial data is in the negative Sobolev space $\dot{H}^{-s}\left(\mathbb{R}^{3}\right)$ with $s \in\left[0, \frac{3}{2}\right)$.

The following lemma plays a key role in the proof of Theorem 1.2. It shows an energy estimate of the solutions in the negative Sobolev space $\dot{H}^{-s}\left(\mathbb{R}^{3}\right)$. Namely, we have

Lemma 4.1. If $\mathcal{E}_{0}:=\left\|u_{0}\right\|_{H^{N}} \ll 1$, for $s \in\left(0, \frac{1}{2}\right]$, we have

$$
\begin{aligned}
& \frac{\mathrm{d}}{\mathrm{d} t} \int\left(\left|\Lambda^{-s} u\right|^{2}+\left|\Lambda^{-s} \nabla u\right|^{2}\right) \mathrm{d} x+\int\left|\nabla \Lambda^{-s} u\right|^{2} \mathrm{~d} x \\
\leq & C\left(\|D u(t)\|_{H^{1}}^{2}+\left\|D^{2} u(t)\right\|_{H^{1}}^{2}\right)\left(\left\|\Lambda^{-s} u(t)\right\|_{L^{2}}+\left\|\Lambda^{-s} \nabla u(t)\right\|_{L^{2}}\right) ;
\end{aligned}
$$

and for $s \in\left(\frac{1}{2}, \frac{3}{2}\right)$, we have

$$
\begin{aligned}
& \frac{\mathrm{d}}{\mathrm{d} t} \int\left(\left|\Lambda^{-s} u\right|^{2}+\left|\Lambda^{-s} \nabla u\right|^{2}\right) \mathrm{d} x+\int\left|\nabla \Lambda^{-s} u\right|^{2} \mathrm{~d} x \\
\leq & C\left(\|u(t)\|_{L^{2}}^{s-\frac{1}{2}}\|D u(t)\|_{L^{2}}^{\frac{5}{2}-s}+\|D u(t)\|_{L^{2}}^{s-\frac{1}{2}}\left\|D^{2} u(t)\right\|_{L^{2}}^{\frac{5}{2}-s}\right)\left(\left\|\Lambda^{-s} u(t)\right\|_{L^{2}}+\left\|\Lambda^{-s} \nabla u(t)\right\|_{L^{2}}\right) .
\end{aligned}
$$


Proof. Applying $\Lambda^{-s}$ to $(2.2)_{1}$ and multiplying the resulting identity by $\Lambda^{-s} u$, and integrating over $\mathbb{R}^{3}$ by parts, we get

$$
\begin{aligned}
& \frac{1}{2} \frac{\mathrm{d}}{\mathrm{d} t} \int\left(\left|\Lambda^{-s} u\right|^{2}+\left|\Lambda^{-s} \nabla u\right|^{2}\right) \mathrm{d} x+\int\left|\nabla \Lambda^{-s} u\right|^{2} \mathrm{~d} x \\
= & -\sum_{j=1}^{3}\left\{\int \Lambda^{-s} u \Lambda^{-s} f_{j}(u)_{x_{j}} \mathrm{~d} x-\int \Lambda^{-s} u \Delta \Lambda^{-s} f_{j}(u)_{x_{j}} \mathrm{~d} x\right\} \\
:= & J_{1}+J_{2} .
\end{aligned}
$$

For $J_{1}$, using Hölder inequality, Lemma 2.1, Lemma 2.3 and Young's inequality, we have

$$
\begin{aligned}
J_{1} & \leq C\left\|\Lambda^{-s} u(t)\right\|_{L^{2}} \sum_{j=1}^{3}\left\|\Lambda^{-s} f_{j}(u)_{x_{j}}(t)\right\|_{L^{2}} \\
& \leq C\left\|\Lambda^{-s} u(t)\right\|_{L^{2}} \sum_{j=1}^{3}\left\|f_{j}(u)_{x_{j}}(t)\right\|_{L^{\frac{1}{2}+\frac{s}{3}}} \leq C\left\|\Lambda^{-s} u(t)\right\|_{L^{2}}\|\nabla u(t)\|_{L^{2}}\|u(t)\|_{L^{\frac{3}{s}}} \\
& \leq C\left\|\Lambda^{-s} u(t)\right\|_{L^{2}}\|\nabla u(t)\|_{L^{2}}\|\nabla u(t)\|_{L^{2}}^{\frac{1}{2}+s}\left\|D^{2} u(t)\right\|_{L^{2}}^{\frac{1}{2}-s} \\
& \leq C\left(\|D u(t)\|_{L^{2}}^{2}+\left\|D^{2} u(t)\right\|_{L^{2}}^{2}\right)\left\|\Lambda^{-s} u(t)\right\|_{L^{2}} .
\end{aligned}
$$

Here we have used the facts $\frac{1}{2}+\frac{s}{3}<1$ and $\frac{3}{s} \geq 6$.

Similarly, it holds that

$$
J_{2} \leq C\left(\left\|D^{2} u(t)\right\|_{L^{2}}^{2}+\left\|D^{3} u(t)\right\|_{L^{2}}^{2}\right)\left\|\Lambda^{-s} \nabla u(t)\right\|_{L^{2}} .
$$

Combining (4.4) and (4.5), we get (4.1).

Next, we want to prove (4.2). A direct calculation as (4.4) gives

$$
\begin{aligned}
J_{1} & \leq C\left\|\Lambda^{-s} u(t)\right\|_{L^{2}} \sum_{j=1}^{3}\left\|\Lambda^{-s} f_{j}(u)_{x_{j}}(t)\right\|_{L^{2}} \\
& \leq C\left\|\Lambda^{-s} u(t)\right\|_{L^{2}} \sum_{j=1}^{3}\left\|f_{j}(u)_{x_{j}}(t)\right\|_{L^{\frac{1}{2}+\frac{s}{3}}} \\
& \leq C\left\|\Lambda^{-s} u(t)\right\|_{L^{2}}\|D u(t)\|_{L^{2}}\|u(t)\|_{L^{\frac{3}{s}}} \leq C\left\|\Lambda^{-s} u(t)\right\|_{L^{2}}\|D u(t)\|_{L^{2}}\|u(t)\|_{L^{2}}^{s-\frac{1}{2}}\|D u(t)\|_{L^{2}}^{\frac{3}{2}-s} \\
& \leq C\|u(t)\|_{L^{2}}^{s-\frac{1}{2}}\|D u(t)\|_{L^{2}}^{\frac{5}{2}-s}\left\|\Lambda^{-s} u(t)\right\|_{L^{2}} .
\end{aligned}
$$

In the same way, one has

$$
I_{2} \leq C\|D u(t)\|_{L^{2}}^{s-\frac{1}{2}}\left\|D^{2} u(t)\right\|_{L^{2}}^{\frac{5}{2}-s}\left\|\Lambda^{-s} u(t)\right\|_{L^{2}} .
$$

Thus we complete the proof of Lemma 4.1.

With Proposition 1.1, Proposition 2.1 and Lemma 4.1 in hand, we are now ready to prove Theorem 1.2.

Proof of Theorem 1.2 for the case of $s \in\left(0, \frac{1}{2}\right]$. Define $\mathcal{E}_{-s}(t)=\left\|\Lambda^{-s} u(t)\right\|_{L^{2}}^{2}+\left\|\Lambda^{-s} \nabla u(t)\right\|_{L^{2}}^{2}$. Integrating (4.1) with respect to $t$, we find for $s \in\left(0, \frac{1}{2}\right]$,

$$
\mathcal{E}_{-s}(t) \leq \mathcal{E}_{-s}(0)+C \int_{0}^{t}\|D u(\tau)\|_{H^{2}}^{2} \sqrt{\mathcal{E}_{-s}(\tau)} \mathrm{d} \tau
$$


From (2.1), we have the estimates of integrability of $\|D u\|_{H^{1}}^{2}$ with respect to $t$. As a result, we have

$$
\mathcal{E}_{-s}(t) \leq \mathcal{E}_{-s}(0)+C \sup _{0 \leq \tau \leq t} \sqrt{\mathcal{E}_{-s}(\tau)} \leq C\left(1+\sup _{0 \leq \tau \leq t} \sqrt{\mathcal{E}_{-s}(\tau)}\right)
$$

This yields $\mathcal{E}_{-s}(t) \leq C_{1}$ with a positive constant $C_{1}$, that is

$$
\left\|\Lambda^{-s} u(t)\right\|_{L^{2}}^{2}+\left\|\Lambda^{-s} q(t)\right\|_{L^{2}}^{2} \leq\left\|\Lambda^{-s} u(t)\right\|_{L^{2}}^{2}+\left\|\Lambda^{-s} \nabla u(t)\right\|_{L^{2}}^{2} \leq C_{1}
$$

This proves (1.8) for $s \in\left(0, \frac{1}{2}\right]$.

Next, we recall the estimate of Lyaponov-type (2.1) as

$$
\frac{\mathrm{d}}{\mathrm{d} t}\left\|D^{l} u(t)\right\|_{H^{1}}^{2}+\left\|D^{l+1} u(t)\right\|_{L^{2}}^{2} \leq 0, \quad 0 \leq l \leq N-1 .
$$

We may use Lemma 2.2 to have

$$
\left\|D^{l+1} u(t)\right\|_{L^{2}} \geq C\left\|\Lambda^{-s} u(t)\right\|_{L^{2}}^{-\frac{1}{l+s}}\left\|D^{l} u(t)\right\|_{L^{2}}^{1+\frac{1}{l+s}} .
$$

From the above inequality and (4.8) we get for each $l$ with $0 \leq l \leq N-1$,

$$
\begin{aligned}
\left\|D^{l+1} u(t)\right\|_{L^{2}}^{2} & \geq \frac{1}{2}\left\|D^{l+1} u(t)\right\|_{L^{2}}^{2}+\frac{1}{2} C C_{1}^{-\frac{1}{l+s}}\left\|D^{l} u(t)\right\|_{L^{2}}^{2\left(1+\frac{1}{l+s}\right)} \\
& \geq C_{2}\left(\left\|D^{l+1} u(t)\right\|_{L^{2}}^{2}+\left\|D^{l} u(t)\right\|_{L^{2}}^{2}\right)^{1+\frac{1}{l+s}}
\end{aligned}
$$

where $C_{2}>0$ is a constant. Thus we deduce the following time differential inequality

$$
\frac{\mathrm{d}}{\mathrm{d} t}\left\|D^{l} u(t)\right\|_{H^{N-l}}^{2}+C_{2}\left(\left\|D^{l} u(t)\right\|_{H^{N-l}}^{2}\right)^{1+\frac{1}{l+s}} \leq 0, \text { for } l=0,1, \cdots, N .
$$

Integrating this inequality, one gets for some constant $C_{3}>0$

$$
\left\|D^{l} u(t)\right\|_{H^{N-l}}^{2} \leq C_{3}(1+t)^{-(l+s)}, \text { for } l=0,1, \cdots, N .
$$

$(2.2)_{2}$ and (4.10) yield

$$
\left\|D^{l} q(t)\right\|_{H^{N-1-l}} \leq C_{4}(1+t)^{-\frac{l+s+1}{2}}, \text { for } l=0, \cdots, N-1 .
$$

Proof of Theorem 1.2 for the case of $s \in\left(\frac{1}{2}, \frac{3}{2}\right)$. First we can give from what we have proved for (1.8)-(1.9) with $s=\frac{1}{2}$ above that the following decay results holds:

$$
\left\|D^{l} u(t)\right\|_{H^{N-l}}^{2} \leq C_{3}(1+t)^{-l-\frac{1}{2}} \text {, for } l=0,1, \cdots, N \text {. }
$$

As a result, and using (4.2) that for $s \in\left(\frac{1}{2}, \frac{3}{2}\right)$,

$$
\begin{aligned}
\mathcal{E}_{-s}(t) & \leq \mathcal{E}_{-s}(0)+C \int_{0}^{t}\|u(\tau)\|_{L^{2}}^{s-\frac{1}{2}}\|D u(\tau)\|_{L^{2}}^{\frac{5}{2}-s} \sqrt{\mathcal{E}_{-s}(\tau)} \mathrm{d} \tau \\
& \leq C_{1}+C C_{3} \int_{0}^{t}(1+\tau)^{-\frac{7}{4}-\frac{s}{2}} \mathrm{~d} \tau \cdot \sup _{0 \leq \tau \leq t} \sqrt{\mathcal{E}_{-s}(\tau)} \\
& \leq C_{5}\left(1+\sup _{0 \leq \tau \leq t} \sqrt{\mathcal{E}_{-s}(\tau)}\right),
\end{aligned}
$$


which yields (1.8) with $s \in\left(\frac{1}{2}, \frac{3}{2}\right)$. At last, the proof of (1.9) with $s \in\left(\frac{1}{2}, \frac{3}{2}\right)$ can be treated as the case of $s \in\left(0, \frac{1}{2}\right]$ above.

Thus, by taking $C_{0}=\max _{1 \leq i \leq 5}\left\{C_{i}\right\}$, we complete the proof of Theorem 1.2.

Acknowledgement: The research of Zhigang Wu was supported by NSFC (No. 11101112) and in part by NSFC (No. 11071162). The research of Wenjun Wang was supported by the Tian Yuan Fund of Mathematics in China (No.11126096), NSFC (No. 11201300), Shanghai university young teacher training program (No. slg11032) and in part by NSFC (No.11171220, No.11171212).

\section{References}

[1] R.J. Duan, K. Fellner and C. Zhu, Energy method for multi-dimensional balance laws with non-local dissipation, J. Math. Pures Appl., 93(6) (2010), 572-598.

[2] M. Di Francesco, Initial value problem and relaxation limits of the Hamer model for radiating gases in several space variables, NoDEA Nonl. Differential Equations Appl., 13 (2007), 531-562.

[3] M. Di Francesco and C. Lattanzio, Optimal $L^{1}$ rate of decay to diffusion waves for the Hamer model of radiating gases, Appl. Math. Lett. 19(10) (2006), 1046-1052.

[4] W.L. Gao, L.Z. Ruan and C.J. Zhu, Decay rates to the planar rarefaction waves for a model system of the radiating gas in n-dimensions, J. Differential Equations, 244 (2008), 2614-2640.

[5] W.L. Gao and C.J. Zhu, Asymptotic decay toward the planar rarefaction waves for a model system of the radiating gas in two dimensions, Math. Models Methods Appl. Sci., 18 (2008), 511-541.

[6] Y. Guo and Y.J. Wang, Decay of dissipative equations and negative Sobolev spaces, arXiv: 1111.5660v1 (2011).

[7] K. Hamer, Nonlinear effects on the propogation of sound waves in a radiating gas, Quart. J. Mech. Appl. Math., 24 (1971), 155-168.

[8] T. Iguchi and S. Kawashima, On space-time decay properties of solutions to hyperbolicelliptic coupled systems, Hiroshima Math. J., 32 (2002), 229-308.

[9] S. Kawashima, Y. Nikkuni and S. Nishibata, The initial value problem for hyperbolicelliptic coupled systems and applications to radiation hydrodynamics, Analysis of Systems of Conservation Laws (H. Freistöehler ed.), Chapman \& Hall/CRC, 1998, 87-127. 
[10] S. Kawashima, Y. Nikkuni and S. Nishibata, Large-time behavior of solutions to hyperbolic-elliptic coupled systems, Arch. Rational Mech. Anal., 170 (2003), 297-329.

[11] S. Kawashima and S. Nishibata, Shock waves for a model system of the radiating gas, SIAM J. Math. Anal., 30 (1998), 95-117.

[12] S. Kawashima and S. Nishibata, Weak solutions with a shock to a model system of the radiating gas, Sci. Bull. Josai. Univ., Special Issue, 5 (1998), 119-130.

[13] S. Kawashima and S. Nishibata, Cauchy problem for a model system of the radiating gas: weak solution with a jump and classical solutions, Math. Models Methods Appl. Sci., 9 (1999), 69-91.

[14] S. Kawashima and S. Nishibata, A singular limit for hyperbolic-elliptic coupled systems in radiation hydrodynamics, Indiana Univ. Math. J., 50 (2001), 567-589.

[15] S. Kawashima and Y. Tanaka, Stability of rarefaction waves for a model system of a radiating gas, Kyushu J. Math., 58 (2004), 211-250.

[16] C. Lattanzio and P. Marcati, Global well-posedness and relaxation limits of a model for radiating gas, J. Differential Equations, 190(2) (2003), 439-465.

[17] C. Lattanzio, C. Mascia, T. Nguyen, R.G. Plaza and K. Zumbrun, Stability of scalar radiative shock profiles, SIAM J. Math. Anal., 41(6) (2009), 2165-2206.

[18] C. Lattanzio, C. Mascia and D. Serre, Shock waves for radiative hyperbolic-elliptic systems. Indiana Univ. Math. J., 56 (2007), 2601-2640.

[19] P. Laurenot, Asymptotic self-similarity for a simplified model for radiating gases, Asymptot. Anal., 42 (2005), 251-262.

[20] C. Lin, J.F. Coulombel and T. Goudon, Asymptotic stability of shock profiles in radiative hydrodynamics. C. R. Math. Acad. Sci. Paris, 345(11) (2007), 625-628.

[21] C. Lin, J.F. Coulombel and T. Goudon, Shock profiles for nonequilibrium radiating gases, Physica D: Nonlinear Phenomena, 218(1) (2006), 83-94.

[22] Y. Liu and S. Kawashima, Asymptotic behavior of solutions to a model system of a radiating gas, Communications on Pure and Applied Analysis, 10 (2011), 209-223.

[23] H. Liu, and E. Tadmor, Critical thresholds in a convolution model for nonlinear conservation laws. SIAM J. Math. Anal., 33(4) (2001), 930-945(electronic).

[24] S. Nishibata, Asymptotic behavior of solutions to a model system of a radiating gas with discontinuous initial data, Math. Models Methods Appl. Sci., 10 (2000), 1209-1231. 
[25] T. Nguyen, R. G. Plaza and K. Zumbrun, Stability of radiative shock profiles for hyperbolic-elliptic coupled systems. Physica D: Nonlinear Phenomena, 239(8) (2010), 428-453.

[26] L.Z. Ruan and C.J. Zhu, Asymptotic behavior of solutions to a hyperbolic-elliptic coupled system in multi-dimensional radiating gas, J. Differential Equations, 249(9) (2010), 2076-2110.

[27] M.E. Schonbek, Decay of solutions to parabolic conservation laws, Comm. Partial Differential Equations, 7 (1980), 449-473.

[28] M.E. Schonbek, Large time behaviour of solutions to the navier-stokes equations, Comm. Partial Differential Equations, 11(7) (1986), 103-117.

[29] M.E. Schonbek, Large time behaviour of solutions to the Navier-Stokes equations in $H^{m}$ space, Comm. Partial Differential Equations, 20 (1995), 733-763.

[30] D. Serre, $L^{1}$-stability of constants in a model for radiating gases, Comm. Math. Sci., 1 (2003), 197-205.

[31] D. Serre, $L^{1}$-stability of nonlinear waves in scalar conservation laws, Evolutionary equations. Vol. I, 473-553, Handb. Differ. Equ., North-Holland, Amsterdam, 2004.

[32] E. M. Stein, Singular Integrals and Differentiability Properties of Functions, Princeton University Press, 1970.

[33] W.K. Wang and W.J. Wang, The pointwise estimates of solutions for a model system of the radiating gas in multi-dimensions, Nonlinear Anal., 71 (2009), 1180-1195. 